\title{
Note on Newton's Theorem of Symmetric Functions.
}

\section{By Charles Tweedie.}

\$. The proofs usually given that $\mathrm{S}_{n}=a^{n}+\beta^{n}+\ldots$ can be expressed in terms of the elementary symmetric functions $\Sigma a, \Sigma \alpha \beta$, etc., though simple, are not in general elementary. The following demonstration will, I think, be found to combine these two qualities.

\$2. If we consider any integral, homogeneous, symmetric function of degree $n$, its expression can be effected by the use of Typical Terms, or Types, and the $\Sigma$ notation. Since these types can not contain more than $n$ letters, it follows that the number of types can not be increased by taking more letters than $n$. Thus the general bomogeneous, symmetric function of the third degree is

$$
A \Sigma \alpha^{3}+B \Sigma \alpha^{2} \beta+C \Sigma \alpha \beta \gamma .
$$

The correct expression for a smaller number of letters may be found by equating a convenient number of the original letters to zero.

It may also be remarked that any identity established among functions of degree $n$ for a number of letters equal to $n$, will also hold for a greater number of letters. For we can not increase the number of types and those which do occur can not have their coefficients altered. (Some of the coefficients may, however, be zero.)

e.gi, $(\Sigma a b)^{2}=\Sigma a^{2} b^{2}+2 \Sigma a^{2} b c+6 \Sigma a b c d$ for four or more letters.

\$3. The ordinary equations for $S_{1}, S_{2}$, etc., may now be readily established.

Let $\Sigma a=-p_{1} ; \Sigma a \beta=p_{2}$; etc., as for the $n$ letters $\alpha, \beta$, etc.

Then the equation

$$
x^{n}+p_{1} x^{n-1}+p_{2} x^{n-2}+\ldots \ldots+p_{n}=0
$$

has for roots $\alpha, \beta, \gamma$, etc. 


\section{1}

Hence

$$
\begin{gathered}
a^{n}+p_{1} a^{n-1}+\ldots \ldots+p_{n}=0, \\
\beta^{n}+p_{1} \beta^{n-1}+\ldots \ldots+p_{n}=0, \\
\text { etc. etc. }
\end{gathered}
$$

Add these $n$ identities and there results

$$
\mathrm{S}_{n}+p_{1} \mathrm{~S}_{n-1}+\ldots \ldots+n p_{n}=0 \text {. }
$$

The same identity is true for any number of letters greater than n. For $n \quad r$ letters put $p_{n-r+1}=0, \ldots \ldots p_{n}=0$.

We thus have the following identities as particular cases, true for $n$ or more letters :

$$
\begin{gathered}
\mathrm{S}_{1}+p_{1}=0 \\
\mathrm{~S}_{2}+p_{1} \mathrm{~S}_{1}+2 p_{2}=0 \\
\cdots \cdots \cdots \cdots \cdots \cdots \cdots \\
\mathrm{S}_{n}+p_{1} \mathrm{~S}_{n-1}+\cdots+n p_{n}=0
\end{gathered}
$$

For three letters put $p_{4}=0, p_{5}=0, \ldots p_{n}=0$, etc. 